\title{
Analizando procesos cognitivos y de escritura en niños hispano-parlantes que aprenden inglés como segunda lengua y niños canadienses de habla inglesa
}

\author{
Isabel O'Shanahan ${ }^{1}$, Linda S. Siegel ${ }^{2}$, Juan E. Jiménez ${ }^{1}$ y Silvia Mazabel ${ }^{2}$ \\ ${ }^{1}$ Universidad de La Laguna (España), ${ }^{2}$ University of British Columbia (Canadá)
}

El objetivo principal de este estudio ha sido analizar los procesos cognitivos y de escritura de niños hispano-parlantes que aprenden el inglés como segunda lengua. Para ello se seleccionó a una muestra de niños canadienses de habla inglesa y otra de hispanoparlantes que reciben instrucción en inglés como segunda lengua en escuelas canadienses del distrito de Vancouver en la provincia canadiense de Columbia Británica (British Columbia). Se tomaron medidas de competencia lingüística (vocabulario y conciencia sintáctica), memoria de trabajo, conciencia fonológica y escritura de palabras y pseudopalabras en inglés para el grupo de monolingües, y las mismas medidas en los idiomas inglés y español para el grupo de hispano-parlantes. Nuestra predicción es que si existe un efecto de transferencia de L1 sobre L2 entonces existiría relación entre los procesos cognitivos y de escritura que se miden en ambas lenguas, y no existirían diferencias significativas en este tipo de medidas que se toman en L2 entre niños canadienses de habla inglesa e hispano-parlante. El principal hallazgo ha sido encontrar que no existen diferencias significativas entre ambos grupos en procesos fonológicos y de escritura de palabras y pseudopalabras, sin embargo, los niños canadienses de habla inglesa presentan mayor competencia lingüística en comparación al grupo de hispanoparlantes ya que obtienen puntuaciones superiores en medidas de vocabulario y de conciencia sintáctica.

Palabras clave: Escritura de palabras, escritura de pseudopalabras, aprendizaje de segunda lengua, competencia lingüística, conciencia fonológica, memoria de trabajo, vocabulario, conciencia sintáctica.

Analyzing cognitive and spelling skills in Spanish-speaking English-language learners and English-speaking Canadian learners. The principal purpose of this study has been to analyze the cognitive processes and spelling skills in Spanish-speaking English-language learners. A sample of English-speaking Canadian learners and Spanish-speaking Englishlanguage learners was selected from different Canadian schools in the Vancouver District within British Columbia's province. We examined cognitive and spelling skills of Englishspeaking students and Spanish-speaking English language learners in the primary grades. We hypothesized that there would be a positive transfer from cognitive and linguistic processes from L1 to L2 spelling skills development, if no significant differences were observed among native English speakers and Spanish-language learners on these measures. There were no significant differences between the English -language learners and the native English speakers on measures of phonological awareness and spelling skills. However, English-speaking Canadian learners performed better than Spanishspeaking English-language learners on vocabulary and syntactic awareness.

Key words: Language-minority students, spelling, English-language learners, oral language proficiency, phonological processes, working memory, vocabulary, syntactic awareness.

Correspondencia: Isabel O'Shanahan. Facultad de Educación. Universidad de La Laguna. Tenerife, Islas Canarias. E-mail: ioshanah@ull.es; ejimenez@ull.es 
El interés por la investigación sobre el aprendizaje de la escritura, en alumnos hispanos-parlantes que aprenden el inglés como segunda lengua, ha ido creciendo en los últimos años tanto en la sociedad norteamericana como canadiense. La mayoría de alumnos bilingües (77\%) hablan el español como primera lengua ${ }^{1}$ (Zehler et al., 2003). No obstante, la proporción de estudios realizados sobre los procesos de escritura en niños que aprenden inglés como segunda lengua ha sido escasa en comparación a los estudios realizados en el ámbito de la lectura (August y Shanahan, 2006).

El estudio de la relación entre las habilidades de escritura entre lengua materna (L1) y segunda lengua (L2) ha estado guiado por dos teorías o hipótesis explicativas: la hipótesis de la interdependencia lingüística (linguistic interdependence hypothesis) (Cummins, 1979) y b) la teoría o hipótesis de la dependencia ortográfica (script-dependent hypothesis) (e.g., Lindgren, DeRenzi, \& Richmand, 1985). La hipótesis de la interdependencia lingüística predice que los niños que tienen problemas en el aprendizaje en su primera lengua también los tendrán en una segunda lengua. Asimismo, predice que las habilidades académicas que se adquieren con éxito en la primera lengua se transfieren a una segunda lengua. La idea principal de esta teoría es que aprender una segunda lengua no impide el progreso de ambas lenguas sino que además puede estimular el desarrollo de las mismas. Por tanto, las dificultades que se puedan tener en una primera lengua también se presentarán en una segunda lengua.

En cambio, la teoría o hipótesis de la dependencia ortográfica establece que las habilidades que se adquieren en una lengua pueden estar influenciadas por su estructura ortográfica y por la predictibilidad de las reglas de conversión fonemagrafema (CFG) (e.g., Lindgren et al., 1985). Entonces, los problemas en la escritura que surjan en una lengua u otra dependerán de las características específicas del sistema ortográfico de la lengua. Así, por ejemplo, mientras que el inglés no tiene una correspondencia directa fonema-grafema, otras lenguas como el español, italiano, etc. tienen reglas de correspondencia mucho más consistentes.

Habilidades críticas para aprender a escribir en inglés como segunda lengua

Una de las habilidades críticas que los niños ELLs deben desarrollar cuando aprenden a escribir en una segunda lengua es la competencia lingüística. Se refiere a las habilidades de comprensión y expresión oral, y también a conocimientos y dominio de la lengua oral, incluyendo habilidades fonológicas, morfológicas, gramaticales, pragmáticas, y vocabulario.

\footnotetext{
${ }^{1}$ En EEUU se conoce a estos alumnos con la denominación ELLs (English Language Learners), en Canadá, ESL (English as a Second Language) y en Reino Unido, EAL (English as an Additional Language). En lo sucesivo utilizaremos las siglas ELLs porque es la que se emplea con mayor frecuencia en la literatura científica.
} 
Otra de las habilidades que tiene una influencia directa y decisiva sobre la adquisición de la escritura es el procesamiento fonológico (e.g., Stanovich \& Siegel, 1994). En general, la investigación ha identificado tres componentes principales del procesamiento fonológico: conciencia fonológica $(\mathrm{CF})$, recodificación fonológica, y memoria fonológica. Se ha sugerido que la mayor dificultad para el reconocimiento de los fonemas en niños que aprenden a escribir se debe a que no constituyen unidades linguiísticas explícitas en la percepción y/o producción del habla. De hecho se ha encontrado una relación entre la conciencia fonológica y la habilidad para escribir grupos consonánticos en los primeros niveles escolares (Jiménez \& Jiménez, 1999). En cuanto a los procesos de memoria, la investigación ha demostrado hasta ahora que la memoria a corto plazo (MCP) y la memoria de trabajo (MT) se relacionan independientemente con la habilidad para escribir las palabras y la planificación del discurso (Swanson \& Siegel, 2001). Durante las primeras etapas de adquisición de la escritura, la MT es crítica para el aprendizaje de las reglas de CFG, ya que cada segmento de la palabra debe ser recordado y matenido en la memoria mientras el escritor codifica cada parte de la palabra (Siegel, 1993).

Las habilidades ortográficas (word-level spelling skills) incluyen una combinación de habilidades fonológicas y ortográficas. En el aprendizaje de la escritura uno de los procesos que más preocupa a los educadores es justamente el que tiene que ver con la ortografía (Jiménez, O’Shanahan, Tabraue et al., 2008). Muchos docentes se quejan de que los alumnos que tienen dificultades ortográficas en los primeros cursos escolares luego continúan con ellas a lo largo de la escolaridad. Estas dificultades con la ortografía pueden afectar a la escritura de diversas formas (Jiménez y Muñetón, 2002). Por ejemplo, una palabra que no esté escrita correctamente puede dificultar al lector la comprensión del mensaje escrito. Escribir incorrectamente también puede influir sobre la percepción del profesorado acerca de la competencia del alumno como escritor. En escritos o redacciones la evaluación de la calidad viene determinada en gran medida por la presencia o no de faltas de ortografía (Marshall y Powers, 1969).También el conocimiento ortográfico influye sobre los procesos de construcción sintáctica y de planificación de la escritura, ya que si estamos prestando mucha atención a cómo se escribe una palabra nos olvidamos de las ideas que estamos organizando y planificando en la memoria de trabajo (Graham, Harris y Fink, 2002).

Por último, la conciencia sintáctica juega también un rol importante en la adquisición de la escritura (Ehri \& Wilce, 1980). La conciencia sintáctica se define como "la habilidad para reflexionar conscientemente sobre los aspectos sintácticos del lenguaje y ejercitar un control intencional sobre la aplicación de reglas gramaticales" (Gombert, 1992, p. 39). Esta habilidad es crítica para la escritura fluida y eficiente del texto, y requiere hacer predicciones acerca de las palabras que estarían dentro de una secuencia. Los factores sintácticos pueden influir en la escritura de palabras funcionales, como 
serían las preposiciones, y verbos auxiliares, los cuales son difíciles de integrar en una red semántica (Siegel, 1992). Para evaluar el conocimiento sintáctico se ha utilizado la tarea Oral cloze que consiste en que los niños escuchan al experimentador leer frases a las que falta una palabra (e.g., "Juan a Pedro una carta hace una semana"). La tarea del niño consiste en encontrar la palabra que permite construir una frase coherente desde el punto de vista sintáctico y semántico (e.g., envió).

Llegados a este punto, los hallazgos hasta ahora aportados por el National Literacy Panel on Language-Minority Children and Youth (August \& Shanahan, 2006) en relación a los procesos cognitivos y de escritura a nivel léxico, sugiere que monolingües y ELLs parecen alcanzar niveles similares de rendimiento en medidas de procesamiento fonológico y escritura de palabras. La mayor parte de estos estudios fueron realizados en diferentes contextos lingüísticos y demográficos (e.g., Canada, Inglaterra, Holanda, EEUU) y con muestras de distintas edades, desde preescolar hasta octavo grado. Los niños ELLs eran nativos de Punjabi, Urdu, Arabe, Italiano, Portugués, y también de distintos contextos lingüísticos del continente asiático. Sin embargo, el número de estudios realizado hasta ahora es escaso en relación a los procesos de escritura en niños hispano-parlantes que aprenden inglés como segunda lengua, y tambien lo ha sido analizar la comparación entre dos sistemas ortográficos que difieren en cuanto a la predictibilidad de las reglas de correspondencia fonema-grafema. En este contexto resulta relevante analizar el efecto de transferencia de los procesos cognitivos y lingüísticos de niños hispano-parlantes sobre los procesos cognitivos y de escritura de la segunda lengua que están aprendiendo (i.e., inglés). Nuestra predicción es que si existe un efecto de transferencia de L1 sobre L2 entonces existiría relación entre los procesos cognitivos y de escritura que se miden en ambas lenguas, y no existirían diferencias significativas en este tipo de medidas que se toman en L2 entre niños canadienses de habla inglesa e hispano-parlante. Asimismo, en este contexto una segunda predicción que se formula es que si se ha aprendido previamente a escribir en una lengua más transparente esto podría también facilitar el desarrollo de procesos cognitivos y de escritura en L2.

\section{METODO}

\section{Participantes}

Se seleccionó una muestra de niños canadienses de habla inglesa $(N=25)$ y niños hispano-parlantes $(N=40)$ escolarizados en escuelas canadienses y que tenían el Inglés como su segunda lengua. Tanto los niños canadienses de habla inglesa como los niños hispano-parlantes fueron seleccionados de $2^{\circ}$ a $7^{\circ}$ grado de Educación Primaria. No se encontraron diferencias significativas entre ambos grupos en edad, $F(1,56)=.04$, $p=$.84. Asimismo, tampoco existían diferencias significativas en la distribución de los 
sujetos según grado y condición lingüística $\chi^{2}(5)=3.75, p=.58$, y tampoco existían diferencias significativas entre condición lingüística y género $\chi^{2}(1)=1.83, p=.17$. En el grupo de niños hispano-parlantes, en cuanto al primer idioma en que aprendieron a escribir, un $30 \%$ lo hizo en inglés, un $42.5 \%$ lo hizo en español, y un $7.5 \%$ lo hizo en ambos idiomas. El $72.5 \%$ se comunica en español con sus padres, y el $2.5 \%$ lo hace en inglés. Sin embargo, con los hermanos, el $30.0 \%$ se comunica en inglés, el $45 \%$ en español, y el 7.5\% en ambos idiomas. En cuanto al país de nacimiento, el $25 \%$ nació en México, el $20 \%$ en Canadá, el $12.5 \%$ en Chile, el $7.5 \%$ en Colombia, el 5\% en Ecuador, el $5 \%$ en el Salvador, el $2.5 \%$ en Argentina, el $2.5 \%$ en Guatemala, y el $2.5 \%$ en Perú. Por tanto, tomados conjuntamente estos datos el $80 \%$ aproximadamente nació fuera de Canadá, y el 20\% nació en Canadá.

\section{Instrumentos}

Pruebas en lengua inglesa

Picture Vocabulary (Vocabulario sobre dibujos). Este test forma parte del Test de habilidades lingüísticas para bilingües (Bilingual Verbal Ability Tests, MuñozSandoval, Cummins, Alvarado \& Ruef, 1998) cuyas pruebas se originan en la batería Woodcock-Johnson-Revised Tests of Cognitive Ability (Woodcock \& Johnson, 1989). En esta prueba se requiere que el sujeto nombre objetos familiares y no familiares a partir de un dibujo. El nivel de dificultad de los ítems aumenta gradualmente. Un total de 58 items conforman la prueba, y el coeficiente de fiabilidad es de .89 .

Oral Cloze Task English (Tarea de conciencia sintáctica) (Siegel \& Ryan, 1988). En esta tarea que evalúa la conciencia sintáctica se presenta oralmente una frase mutilada y el niño debe decir una palabra que la complete sintáctica y semánticamente de manera correcta. Un total de 20 frases es presentado y se asigna un punto por cada frase resuelta correctamente. Un ejemplo de frase mutilada es: Jane___ her sister ran up the hill. El coeficiente de fiabilidad es de .87

Working Memory (Memoria de trabajo). Se utilizó la prueba original en inglés que adaptaron Siegel y Ryan (1989) siguiendo el procedimiento de Daneman y Carpenter (1980) y que consiste en presentar al niño grupos de 2, 3, 4 ó 5 frases que no contienen la última palabra. En esta tarea el niño debe emitir una palabra que complete cada frase presentada y luego decir en el orden correcto todas las palabras emitidas. Se asigna un punto por cada grupo de palabras recordadas en el orden correcto para cada grupo de frases. Hay 3 ensayos para cada grupo de frases y se asigna un punto por cada serie conseguida.

Phonemic Awareness Task (Tarea de conciencia fonológica). Para evaluar la conciencia fonológica en inglés se hizo una adaptación de la tarea de omisión de fonemas que está incluida en la Batería Multimedia Sicole-R-Primaria (Jiménez et al., 2007) en la cual el niño escucha una palabra y debe responder diciendo cómo quedaría la 
palabra si eliminásemos el fonema inicial (v.gr. se escucha /flight/ la respuesta correcta sería /light/). Un total de 10 palabras fueron presentadas. El coeficiente de fiabilidad para esta tarea es .83.

Wide Range Achievement Test-3 (WRAT-3; Wikilson, 1993). Se utilizó la subprueba de Spelling que consiste en la escritura al dictado de algunas letras del alfabeto y de un máximo de 40 palabras sin límite de tiempo. El sujeto primero escucha la palabra aislada, después en contexto y nuevamente de manera aislada. Se asigna un punto por cada palabra escrita correctamente. El coeficiente de fiabilidad es de .91.

Spelling of Sounds. Esta es una prueba de la Batería de Rendimiento Academico Woodcock-Johnson III (WJ III ${ }^{\circledR}$ Tests of Achievement, Woodcock, McGrew, \& Mather, 2001) que consiste en escribir pseudopalabras que se ajustan a las reglas de escritura del ingles. Esta prueba evalúa tanto aspectos fonológicos como ortográficos de la codificación escrita. Un máximo de 28 pseudopalabras es dictado al sujeto. Se hizo una modificación respecto al procedimiento estandarizado de administracion de esta prueba ya que los estímulos fueron dictados por los evaluadores y no se utilizó la grabación. El coeficiente de fiabilidad es de .76.

Pruebas en lengua española

Test de habilidades lingüísticas para bilingües (Bilingual Verbal Ability Test) (Muñoz-Sandoval et al., 1998). Una descripción de este test ha sido presentada en la sección donde describimos los materiales empleados en inglés. En este caso utilizamos la versión en español de la prueba vocabulario basado en imágenes. El procedimiento estándar de administración fue alterado ya que en este caso presentamos a cada sujeto un máximo de 58 items y no únicamente aquellos que no pudo nombrar en inglés.

Tarea de conciencia sintáctica (Oral Cloze Task Spanish) (Jiménez, Mazabel, O'Shanahan \& Siegel, 2008). En esta tarea el niño tiene debe decir la palabra que falta en una serie de 20 frases que se presentan oralmente. Un ejemplo de frase mutilada es: Beatriz___varios agujeros con la pala. Se asigna un punto por frase resuelta correctamente.

Conciencia fonológica (Phonemic Awareness Test). Se utilizó la tarea de omisión de fonemas que está incluida en la Batería Multimedia Sicole-R-Primaria (Jiménez et al., 2007). La tarea de omitir fonemas consiste en escuchar una palabra emitida desde el ordenador y el niño debe responder diciendo cómo quedaría la palabra si eliminásemos el fonema inicial (v.gr. se escucha /lata/ la respuesta correcta sería /ata/).

Memoria de Trabajo (Working memory). Esta prueba consiste en una adaptación al español de la tarea de Siegel y Ryan (1989), y desarrollada a través del procedimiento propuesto por Daneman y Carpenter (1980).

Proescri-Primaria (Prueba de Evaluación de los Procesos Cognitivos en la Escritura) (Artiles y Jiménez, 2007). Esta prueba comprende un total de 15 tareas de 
diferente complejidad. Para la evaluación de los procesos léxicos hemos administrado solamente las tareas de escritura al dictado de palabras y pseudopalabras. Las tareas administradas han sido: 1) dictado de 20 palabras con distinta longitud y familiaridad, y 2) dictado de 20 pseudopalabras con distinta longitud y frecuencia silábica posicional. El coeficiente de fiabilidad de esta prueba es de .85 .

\section{Procedimiento}

Para la realización de este trabajo se siguieron las normas establecidas por la provincia canadiense de Columbia Británica (Bristish Columbia) para la investigación con escolares, que exige la aprobación del proyecto por parte del Comité de Ética de la Universidad de Columbia Británica (University of British Columbia, UBC) y, posteriormente, la aprobación del Consejo Escolar del Distrito de Vancouver (Vancouver School Board, VSB). Una vez conseguidos todos los permisos oficiales se contactó con el Centro de Recepción y Ubicación del Distrito (Discrict Reception and Placement Centre, DRPC) que nos facilitó información sobre la población hispanoparlante escolarizada en el distrito de Vancouver. Asimismo, para acceder a esta población fue necesario contactar a los Consejeros Multiculturales (Multicultural Liaison Workers) y a los Servicios de Adaptación e Integración a la sociedad canadiense para familias inmigrantes y refugiados (Settlement Workers in Schools, SWIS). Los Consejeros multiculturales son parte del personal de recursos culturales del Consejo Escolar del Distrito de Vancouver. De esta forma se pudo contar con la participación de 5 colegios: Hill University Elementary School, Trudeau Elementary School, Moberly Elementary School, Laura Secord Elementary School, y Thunderbird Elementary School. Todos los niños fueron evaluados por examinadores nativos en su propia lengua, de forma que las pruebas en inglés fueron administradas por examinadores canadienses de habla inglesa a los grupos canadiense e hispano-parlante, y los examinadores hispanos administraron las pruebas en español al grupo hispano-parlante. Asimismo, se contrabalanceo el orden de presentación de las pruebas de inglés y español a los distintos grupos con el fin de controlar el efecto debido al orden de presentación.

\section{RESULTADOS}

\section{Correlaciones entre tareas en L1 y L2}

La tabla 1 recoge las correlaciones entre las puntuaciones obtenidas por el grupo de hispano-parlantes sobre las tareas en L1 y L2. Entre las tareas que miden las mismas habilidades en ambos idiomas, se puede observar que existen correlaciones significativas entre la escritura de palabras y pseudopalabras y conciencia fonológica, lo cual indica un rendimiento similar en ambas lenguas. En cambio, no hubo correlaciones 
significativas entre las medidas de vocabulario, memoria de trabajo y conciencia sintáctica entre ambas lenguas.

Tabla 1. Correlaciones entre las medidas de inglés y español en procesos cognitivos y de escritura en niños hispano-parlantes

\begin{tabular}{|c|c|c|c|c|c|c|}
\hline \multirow[b]{2}{*}{ Medidas en inglés } & \multicolumn{6}{|c|}{ Medidas en español } \\
\hline & $\begin{array}{l}\text { Vocabulario } \\
\text { en español }\end{array}$ & $\begin{array}{l}\text { Conciencia } \\
\text { sintáctica en } \\
\text { español }\end{array}$ & $\begin{array}{c}\text { Memoria } \\
\text { de trabajo } \\
\text { en español }\end{array}$ & $\begin{array}{c}\text { Conciencia } \\
\text { fonológica en } \\
\text { español }\end{array}$ & $\begin{array}{c}\text { Escritura de } \\
\text { palabras en } \\
\text { español }\end{array}$ & $\begin{array}{c}\text { Escritura de } \\
\text { pseudopalabras } \\
\text { en español }\end{array}$ \\
\hline Vocabulario & .12 & .16 & .06 & .30 & -.02 & .06 \\
\hline Conciencia sintáctica & -.08 & .23 & .12 & .20 & -.08 & -.01 \\
\hline Memoria de trabajo & .02 & .26 & .18 & .21 & -.04 & .11 \\
\hline Conciencia fonológica & .19 & $.37(*)$ & .16 & $.61(* *)$ & $.39(*)$ & $.38(*)$ \\
\hline Escritura de palabras & .32 & $.39(*)$ & $.40(*)$ & $.63(* *)$ & $.39(*)$ & $.40(*)$ \\
\hline Escritura de pseudopalabras & .11 & .20 & .20 & $.51(* *)$ & .25 & .29 \\
\hline
\end{tabular}

** La correlación es significativa al nivel 0.01 (bilateral).

* La correlación es significante al nivel 0.05 (bilateral).

La tabla 2 muestra las correlaciones obtenidas entre las tareas utilizadas solo en L2 en el grupo de niños canadienses de habla inglesa, y la tabla 3 las correlaciones entre las tareas utilizadas sólo en L1 para la muestra de hispano-parlantes.

Tabla 2. Correlaciones entre las medidas de inglés en niños canadienses de habla inglesa

\begin{tabular}{|c|c|c|c|c|c|c|}
\hline & 1 & 2 & 3 & 4 & 5 & 6 \\
\hline 1. Vocabulario & 1.00 & $.78(* *)$ & $.61(* *)$ & .29 & $.69(* *)$ & $.66(* *)$ \\
\hline 2. Conciencia sintáctica & & 1.00 & $.70(* *)$ & $.39(*)$ & $.60(* *)$ & $.64(* *)$ \\
\hline 3. Memoria de trabajo & & & 1.00 & $.39(*)$ & $.43(* *)$ & $.36(*)$ \\
\hline 4. Conciencia fonológica & & & & 1.00 & $.51(* *)$ & $.56(* *)$ \\
\hline 5. Escritura de palabras & & & & & 1.00 & $.83(* *)$ \\
\hline 6. Escritura de pseudopalabras & & & & & & 1.00 \\
\hline
\end{tabular}

Tabla 3. Correlaciones entre las medidas de español en niños hispano-parlantes

\begin{tabular}{|c|c|c|c|c|c|c|}
\hline & 1 & 2 & 3 & 4 & 5 & 6 \\
\hline 1. Vocabulario & 1.00 & $.57(* *)$ & $.53(* *)$ & $.47(* *)$ & $.76(* *)$ & $.69(* *)$ \\
\hline 2. Conciencia sintáctica & & 1.00 & $.71(* *)$ & $.52(* *)$ & $.58(* *)$ & $.60(* *)$ \\
\hline 3. Memoria de trabajo & & & 1.00 & $.47(* *)$ & $.61(* *)$ & $.54(* *)$ \\
\hline 4. Conciencia fonológica & & & & 1.00 & $.69(* *)$ & $.70(* *)$ \\
\hline 5. Escritura de palabras & & & & & 1.00 & $.88(* *)$ \\
\hline 6. Escritura de pseudopalabras & & & & & & 1.00 \\
\hline
\end{tabular}

Se puede observar que existen correlaciones significativas entre todas las tareas que miden los procesos cognitivos y de escritura en inglés, excepto entre conciencia fonológica y vocabulario. En el caso del español, existen correlaciones significativas entre todas las tareas que miden los procesos cognitivos y de escritura. 


\section{Procesos cognitivos y de escritura en L2}

Con el fin de analizar si existe un efecto de transferencia de L1 a L2, llevamos a cabo un MANOVA mediante modelo lineal general con variable independiente intersujeto: condición lingüística (i.e., monolingüe vs. ELLs) y como variables dependientes: escritura de palabras, escritura de pseudopalabras, conciencia sintáctica, memoria de trabajo, vocabulario y conciencia fonológica en L2. Los resultados mostraron que la condición lingüística tiene una influencia sobre las variables medidas $F(6,56)=3.60, p<.004, \eta^{2}=27$. Con el fin de averiguar en qué procesos cognitivos específicos y de escritura existían diferencias significativas entre los grupos, se realizaron contrastes univariados para cada una de las variables medidas, escritura de palabras $F(1,61)=.55, p=.46, \eta^{2}=009$; escritura de pseudopalabras $F(1,61)=3.40$, $p=.07, \eta^{2}=.05$; conciencia sintáctica $F(1,61)=9.98, p=.002, \eta^{2}=.14$; memoria de trabajo $F(1,61)=3.29, p=.07, \eta^{2}=.05$; vocabulario $F(1,61)=12.8, p<.001, \eta^{2}=.17 ; \mathrm{y}$ conciencia fonológica $F(1,61)=3.53, p=.06, \eta^{2}=.05$. Esto significa que no existían diferencias significativas entre los grupos en escritura de palabras y pseudopalabras, memoria de trabajo y conciencia fonológica. En cambio, los niños canadienses de habla inglesa obtienen mejores puntuaciones en vocabulario y conciencia sintáctica en comparación a los niños hispano-parlantes. La tabla 4 recoge las medias y desviaciones típicas en las medidas en lengua inglesa para ambos grupos.

Tabla 4. Medias y desviaciones típicas en tareas que miden procesos cognitivos y de escritura en lengua inglesa en niños canadienses de habla inglesa e hispano-parlante

\begin{tabular}{llcrr}
\hline & & & Grupo & \\
\cline { 3 - 5 } & & $\begin{array}{c}\text { Niños canadienses } \\
\text { de habla inglesa }\end{array}$ & $\begin{array}{c}\text { Niños } \\
\text { hispano-parlantes }\end{array}$ & Total \\
\hline \multirow{2}{*}{ Vocabulario } & $\mathrm{M}$ & 33.88 & 28.68 & 30.77 \\
& $\mathrm{DT}$ & 4.13 & 5.83 & 5.78 \\
\hline \multirow{2}{*}{ Conciencia sintáctica } & $\mathrm{M}$ & 15.48 & 11.89 & 13.34 \\
& $\mathrm{DT}$ & 3.51 & 5.05 & 3.80 \\
\hline \multirow{2}{*}{ Memoria de trabajo } & $\mathrm{M}$ & 3.72 & 3.16 & 1.23 \\
\hline \multirow{2}{*}{ Conciencia fonológica } & $\mathrm{DT}$ & 1.14 & 1.26 & 8.58 \\
& $\mathrm{M}$ & 9.04 & 1.27 & 29.73 \\
\hline \multirow{2}{*}{ Escritura de palabras } & $\mathrm{DT}$ & 1.49 & 28.54 & 7.27 \\
\hline \multirow{2}{*}{ Escritura de pseudopalabras } & $\mathrm{M}$ & 31.48 & 7.32 & 32.37 \\
& $\mathrm{DT}$ & 6.96 & 30.76 & 6.69 \\
\hline
\end{tabular}

Por otra parte, en el análisis anterior no tuvimos en cuenta si el hecho de haber aprendido primero a escribir en español, esto es, en una ortografía más transparente que 
el inglés podría influir para que los niños hispano-parlantes alcancen un mejor rendimiento en las tareas que miden los procesos cognitivos y de escritura asociados a L2. Los resultados demuestran de nuevo un efecto de la condición lingüística $F(6,40)=$ 8.72, $p<.000, \eta^{2}=.56$. Los contrastes univariados arrojaron los siguientes resultados: escritura de palabras $F(1,45)=.01, p=.92, \eta^{2}=.000$; escritura de pseudopalabras $F(1$, $45)=1.82, p=.18, \eta^{2}=.03$; conciencia sintáctica $F(1,45)=11.3, p=.002, \eta^{2}=.20$; memoria de trabajo $F(1,45)=3.84, p=.056, \eta^{2}=.07$; vocabulario $F(1,45)=23.7, p<.001$, $\eta^{2}=.34$; y conciencia fonológica $F(1,45)=.72, p=.40, \eta^{2}=.01$. Como se puede observar las diferencias encontradas van en la misma dirección, esto es, no existen diferencias significativas entre los grupos en escritura de palabras y pseudopalabras, memoria de trabajo y conciencia fonológica, en cambio, sí existen diferencias en competencia lingüística (i.e., vocabulario y conciencia sintáctica) a favor del grupo de niños canadienses de habla inglesa. La tabla 5 recoge las medias y desviaciones típicas en las medidas en lengua inglesa para los niños canadienses de habla inglesa y los niños hispano-parlantes que aprendieron a escribir primero en español.

Tabla 5. Medias y desviaciones típicas en tareas que miden procesos cognitivos y de escritura en lengua inglesa en niños canadienses de habla inglesa e hispano-parlante que aprendieron a escribir primero en español

\begin{tabular}{llrrr} 
& & \multicolumn{3}{c}{ Grupo } \\
\cline { 3 - 5 } & & $\begin{array}{c}\text { Niños canadienses } \\
\text { de habla inglesa }\end{array}$ & $\begin{array}{c}\text { Niños hispano- } \\
\text { parlantes instruidos } \\
\text { primero en español }\end{array}$ & Total \\
\hline \multirow{2}{*}{ Vocabulario } & M & 33.88 & 26.64 & 30.49 \\
& DT & 4.13 & 5.99 & 6.21 \\
\hline \multirow{2}{*}{ Conciencia sintáctica } & $\mathrm{M}$ & 15.48 & 10.86 & 13.32 \\
& $\mathrm{DT}$ & 3.51 & 5.73 & 5.18 \\
\hline \multirow{2}{*}{ Memoria de trabajo } & $\mathrm{M}$ & 3.72 & 3.00 & 3.38 \\
& $\mathrm{DT}$ & 1.14 & 1.38 & 8.87 \\
\hline \multirow{2}{*}{ Escritura de palabras } & $\mathrm{M}$ & 9.04 & 8.68 & 1.44 \\
\hline \multirow{2}{*}{ Escritura de pseudopalabras } & $\mathrm{DT}$ & 1.49 & 1.39 & 30.45 \\
& $\mathrm{M}$ & 31.48 & 8.47 & 7.70 \\
\hline
\end{tabular}

\section{DISCUSION}

Una primera aproximación a los datos obtenidos nos permiten constatar que tanto la escritura de palabras como de pseudopalabras en L1 y L2 están significativamente relacionadas, así como la conciencia fonológica medida en ambas lenguas. Respecto a esta última, este resultado es coincidente con aquellos estudios que han encontrado también correlaciones significativas cuando se ha analizado la relación 
entre medidas de conciencia fonológica en L1 y L2 en niños que aprenden una segunda lengua (Abu-Rabia, 1997; Cisero \& Royer, 1995; Gottardo, 2002; Gottardo, Yan, Siegel \& Wade-Woolley, 2001; Hsia, 1992; Mumtaz \& Humphreys, 2001; Quiroga, LemosBritten, Mostafapour, Abbott \& Berninger, 2002). En cambio, son pocos los estudios que hayan analizado la relación entre medidas de procesamiento fonológico en L1 con las habilidades ortográficas en L2 (Geva, 2006). En la presente investigación la conciencia fonológica en L1 sí mantiene relación con las habilidades ortográficas en L2 lo cual es coincidente con algunos estudios que analizaron en español la relación entre conciencia fonológica y habilidades ortográficas en inglés (v.gr., Cronnell, 1985; Ferroli y Shanahan, 1993).

Sin embargo, las medidas de lenguaje oral (i.e., vocabulario y conciencia sintáctica) y memoria de trabajo en L1 y L2 no correlacionan entre sí de forma significativa. En general, esta relación sólo se observa cuando se utilizan medidas de vocabulario que no están basadas en definiciones simples sino complejas o categoriales (Carlisle, Beeman, Davis y Spharim, 1999; Johnson, 1989; Ordoñez, Carlo, Snow y McLaughlin, 2002).

Cuando hemos analizado si existen diferencias entre los niños canadienses de habla inglesa e hispano-parlantes en los procesos léxicos (i.e., escritura de palabras y pseudopalabras), no se encontraron diferencias significativas, lo que demuestra el efecto de transferencia de una lengua sobre la otra. Esta equivalencia en los procesos léxicos entre ambos grupos se ha encontrado en distintos estudios realizados en distintos contextos (i.e., Canadá, Reino Unido, Holanda, Estados Unidos) que han incluido diferentes grupos de edades (desde preescolar hasta la ESO), diferentes niveles de habilidad; y donde se ha empleado en unos casos un diseño longitudinal y en otros un diseño de tipo transversal. Asimismo, estos estudios han incluido muestras de niños que aprendían inglés como segunda lengua y tenían como lengua materna el Arabe (AbuRabia \& Siegel, 2002), Italiano (D`Angiulli, Siegel \& Serra, 2001), Portugués (Da Fontoura \& Siegel, 1995); y distintos contextos plurilingües o mixtos (Wade-Wooley \& Siegel, 1997). Sin embargo, otros estudios encontraron que las habilidades ortográficas no estaban tan avanzadas como en los monolingües (v.gr., Fashola, Drum, Mayer y Kang, 1996; Verhoeven, 2000).

A pesar de estos resultados divergentes, lo que parece más consistente es que existen diferencias entre ambos grupos en medidas de competencia lingüística (v.gr., vocabulario y conciencia sintáctica). De hecho, hay una serie de estudios que han informado acerca de diferencias significativas en conciencia sintáctica entre niños monolingües de habla inglesa y ELLs (Chiappe et al., 2002; Lesaux \& Siegel, 2003; Wade-Woolley \& Siegel, 1997). En la mayoría de estos estudios los niños monolingües obtienen puntuaciones superiores a los niños ELLs en las tareas de Oral cloze a pesar de no existir diferencias entre ellos cuando son comparados en tareas de escritura de 
palabras o pseudopalabras. Esto último se ha constatado en el estudio de Wade-Woolley y Siegel (1997) que incluían una muestra de niños ELLs de distintas lenguas como el Cantonés, Mandarin, Guajarati, Urdu, Punjabi y Portugués. Pero también se han encontrado estos resultados en los pocos estudios realizados en Canadá que han examinado contextos idiomáticos específicos (e.g., en Punjabi; Chiappe \& Siegel, 1999; en Portugués (Da Fontoura \& Siegel, 1995). Sin embargo, un patrón de resultados diferentes se ha dado en árabe (Abu-Rabia \& Siegel, 2002) e italiano (D'Angiulli, Siegel \& Serra, 2001). Tanto en el caso de los niños ELLs árabes como portugueses no se encontraron diferencias significativas. Estos hallazgos sugieren que la adquisición o desarrollo de la conciencia sintáctica parece variar en función de los diferentes contextos idiomáticos.

En síntesis, el hecho de que el desarrollo en estas habilidades lingüísticas en L2 sea superior en los niños canadienses de habla inglesa que en los niños hispanoparlantes no implica que el desarrollo de las habilidades ortográficas de este segundo grupo en la segunda lengua se vea comprometido. Por último, también se ha sugerido que aprender a escribir previamente en una lengua que presenta una mayor transparencia ortográfica puede facilitar el desarrollo de los procesos léxicos en L2 cuando ésta presenta una ortografía opaca. En nuestro estudio, los hallazgos encontrados nos permiten sugerir que el aprender a escribir en español antes o después de hacerlo en inglés no parece tener una influencia decisiva para aprender a escribir esta última lengua, ya que no se encuentran diferencias significativas entre los niños hispano-parlantes en función de si aprendieron a escribir primero en español o en inglés.

En general, los hallazgos encontrados en esta investigación parecen apoyar la teoría de la interdependencia lingüística. Se ha podido demostrar que las habilidades de escritura en ambas lenguas están estrechamente relacionadas y nos existen diferencias significativas entre los grupos en el desarrollo de procesos fonológicos y de las habilidades ortográficas en la escritura.

\section{Agradecimientos}

Queremos agradecer especialmente la colaboración prestada por el profesorado del período de Educación Primaria de los centros pertenecientes a la muestra del presente estudio, Hill University Elementary School, Trudeau Elementary School, Moberly Elementary School, Laura Secord Elementary School, y Thunderbird Elementary School, así como a sus respectivos Equipos Directivos y Servicio de Inspección Educativa. También al excelente trabajo realizado por las examinadoras Sarah Husain, Jessica Trach, Kathryn Thompson, Karen Stewart, Shindy Pletzer, Janine M. Simpson y Marcela Alejandra Vidal Vega. Algunas partes de este artículo fueron redactadas mientras el primer y tercer autor estaban como profesores visitantes en el Department of Educational and Counselling Psychology and Special Education en la 
University of British Columbia de Canadá, gracias a la ayuda de movilidad concedida por el Ministerio de Educación y Ciencia de España con ref. PR2007-0405 y PR20070395 respectivamente. Asimismo, esta investigación ha recibido financiación del Natural Sciences and Engineering Research Council of Canada and the Canadian Language and Literacy Research Network del que es IP la segunda autora.

\section{REFERENCIAS}

Abu-Rabia, S. (1997). Verbal and working memory skills of bilingual Hebrew-English speaking children. International Journal of Psycholinguistics, 13(1), 25-40.

Abu-Rabia, S. y Siegel, L.S. (2002). Reading, syntactic, orthographic, and working memory skills of bilingual Arabic-English speaking Canadian children. Journal of Psycholinguistic Research in Reading, 21, 201-212.

Arab-Moghaddam, N. \& Sénéchal, M. (2001). Orthographic and phonological processing skills in reading and spelling in Persian/English bilinguals. International Journal of Behavioral Development, 25, 140-147.

Artiles, C. y Jiménez, J.E. (2007). Proescri Primaria. Prueba de evaluación de procesos cognitivos en la escritura. Islas Canarias: Consejería de Educación, Cultura y Deportes del Gobierno de Canarias.

August, D. y Shanahan, T. (2006). Developing literacy in second-language learners: Report of the national literacy panel on language-minority children and youth. Mahwah, NJ: Lawrence Erlbaum Associates.

Caravolas, M. (2004). Spelling development in alphabetic writing systems: A cross-linguistic perspective. European Psychologist, 9(1), 3-14.

Carlisle, J.F., Beeman, M.M., Davis, L.H. y Spharim, G. (1999). Relationship of metalinguistic capabilities and reading achievement for children who are becoming bilingual. Applied Psycholinguistics, 20(4), 459-478.

Chiappe, P. y Siegel, L.S. (1999). Phonological awareness and reading acquisition in English and Punjabi-speaking Canadian children. Journal of Educational Psychology, 91(1), 20-28.

Chiappe, P., Siegel, L.S. y Gottardo, A. (2002). Reading-related skills of kindergartens from diverse linguistic backgrounds. Applied Psycholinguistics, 23, 95-116.

Chiappe, P., Siegel, L.S. y Wade-Woolley, L. (2002). Linguistic diversity and the development of reading skills: A longitudinal study. Scientific Studies of Reading, 6, 369-400.

Cisero, C.A. y Royer, J.M. (1995). The development and cross-language transfer of phonological awareness. Contemporary Educational Psychology, 20, 275-303.

Cronnell, B. (1985). Language influences in the English writing of third-and sixth-grade Mexican American students. Journal of Educational Research, 78(3), 168-173.

Cummins, J. (1979). Linguistic interdependence and the educational development of bilingual children. Review of Educational Research, 49(2), 222-251.

D'Angiulli, A., Siegel, L.S. y Serra, E. (2001). The development of reading in English and Italian in bilingual children. Applied Pycholinguistics, 22, 479-507.

Da Fontoura, H.A. y Siegel, L.S. (1995). Reading, syntactic, and working memory skills of bilingual Portuguese-English Canadian children. Reading and Writing, 7, 139-153.

Daneman, M. y Carpenter, P.A. (1980). Individual differences in working memory and reading. Journal of Verbal Learning and Verbal Behaviour, 19(4), 450-466.

Ehri, L.C. y Wilce, L.S. (1980). The influence of orthograph on readers' conceptualization of the phonemic structure of words. Applied Psycholinguistics, 1, 371-385. 
Fashola, O.S., Drum, P.A., Mayer, R.E. y Kang, S.J. (1996). A cognitive theory of orthographic transitioning: Predictable errors in how Spanish-speaking children spell English words. American Educational Research Journal, 33(4), 825-843.

Ferroli, L. y Shanahan, T. (1993). Voicing in Spanish to English knowledge transfer. Yearbook of the National Reading Conference, 42, 413-418.

Geva, E. (2006). Second-language oral proficiency and second-language literacy. En D. August y T. Shanahan (Eds.). Developing literacy in second-language learners: Report of the national literacy panel on language-minority children and youth. (pp. 123-139). Mahwah, NJ: Lawrence Erlbaum Associates.

Gombert, J. (1992). Metalinguistic development. París: Chicago University Press.

Gottardo, A. (2002). The relationship between language and reading skills in bilingual SpanishEnglish speakers. Topics in Language Disorders, 22(5), 46-70.

Gottardo, A., Yan, B., Siegel, L.S. y Wade-Woolley, L. (2001). Factors related to English reading performance in children with Chinese as a first language: More evidence of crosslanguage transfer of phonological processing. Journal of Educational Psychology, 93(3), $530-542$.

Graham, S., Harris, K. y Fink, B. (2002). Contribution of spelling instruction to the spelling, writing, and reading of poor spellers. Journal of Educational Psychology, 94, 669-686.

Hsia, S. (1992). Developmental knowledge of inter- and intraword boundaries: Evidence from American and Mandarin Chinese speaking beginning readers. Applied Psycholinguistics, 13, 341-372.

Jiménez, J.E., Antón, L., Diaz, A., Estévez, A., García, A.I., García, E., Guzmán, R., HernándezValle, I., Ortiz, M. R. y Rodrigo, M. (2007). Sicole-R-Primaria: Un sistema de evaluación de los procesos cognitivos en la dislexia mediante ayuda asistida a través del ordenador [Software informático]. Universidad de La Laguna: Autores.

Jiménez, J.E., \& Jiménez, J.E. (1999). Errores en la escritura de sílabas con grupos consonánticos: un estudio transversal. Psicothema, 11(1), 125-135.

Jiménez, J.E., Mazabel, S., O’Shanahan, I. y Siegel, L.S. (2009). Test de conciencia sintáctica. Test no publicado.

Jiménez, J. E. y Muñetón, M. A. (2002).Dificultades de aprendizaje de la escritura. Aplicaciones de la Psicolingüística y de las nuevas tecnologías. Madrid: Trotta.

Jiménez, J.E., O’Shanahan, I., Tabraue el Jaber, M., Artiles, C., Muñetón, M., Guzmán, R., Naranjo. F., y Rojas, E. (2008). Evolución de la escritura de palabras de ortografía arbitraria en lengua española. Psicothema, 20(4), 786-794.

Johnson, J. (1989). Factors related to cross-language transfer and metaphor interpretation of bilingual children. Applied Psycholinguistics, 10, 157-177.

Lesaux, N.K. y Siegel, L.S. (2003). The development of reading in children who speak English as a second language. Developmental Psychology, 39(6), 1005-1019.

Lindgren, S.D., De Renzi, E. y Richman, L.C. (1985). Cross-national comparisons of developmental dyslexia in Italy and the United States. Child Development, 56, 14041417.

Marshall, J. y Powers, J. (1969). Writing neatness, composition errors, and essay grades. Journal of Educational Measurement, 6, 97-101.

Mumtaz, S. y Humphreys, G.W. (2001). The effects of bilingualism on learning to read English: Evidence from the contrast between Urdu-English bilingual and English monolingual children. Journal of Research in Reading, 24(2), 113-134.

Muñoz-Sandoval, A.F., Cummins, J., Alvarado, C.G. y Ruef, M.L. (1998). Bilingual verbal ability tests: Comprehensive manual. Itasca, IL: Riverside. 
Ordoñez, C.L., Carlo, M.S., Snow, C.E. y McLaughlin, B. (2002). Depth and breadth of vocabulary in two languages: Which vocabulary skills transfer? Journal of Educational Psychology, 94(4), 719-728.

Quiroga, T., Lemos-Britton, Z., Mostafapour, E., Abbott, R.D. y Berninger, V.W. (2002). Phonological awareness and beginning reading in Spanish-speaking ESL first graders: Research into practice. Journal of School Psychology, 40(1), 85-111.

Siegel, L.S. (1992). An evaluation of the discrepancy definition of dyslexia. Journal of Learning Disabilities, 25(10), 618-629.

Siegel, L.S. (1993). The development of reading. En H.W. Reese (Ed.), Advances in child development and behavior (pp. 63-97). San Diego: Academic Press.

Siegel, L.S. y Ryan, E.B. (1988). Development of sensitivity, phonological and short-term memory skills in normally achieving and learning disabled children. Developmental Psychology, 24, 28-37.

Siegel, L.S. y Ryan, E.B. (1989). The development of working memory in normally achieving and subtypes of learning disabled children. Child Development, 60, 973-980.

Stanovich, K.E. y Siegel, L.S. (1994). Phenotypic performance profile of children with reading disabilities: A regression-based test of the phonological-core variable-difference model. Journal of Educational Psychology, 86, 24-53.

Swanson, H.L. y Sáez, L. (2003). Memory difficulties in children and adults with learning disabilities. En H.L. Swanson, K. Harris y S. Graham (Eds.), Handbook of learning disabilities (pp. 182-198). New York: Guilford.

Swanson H.L. y Siegel L. (2001). Learning disabilities as a working memory deficit Issues in education: Contributions from educational psychology, 7(1), 1-48.

Treiman, R. y Zukowsky, A. (1991). Levels of phonological awareness. En Brady, S.A. \& Shankweiler, D. (Eds). Phonological processes in literacy. A tribute to Isabelle Y. Liberman (pp. 67-83). Hillsdale, N.Y. Lawrence Erlbaum Associates.

Verhoeven, L.T. (2000). Components in early second language reading and spelling. Scientific Studies of Reading, 4(4), 313-330.

Zehler, A.M., Fleischman, H.L., Hopstock, P.J., Stephenson, T.G., Pendzick, M. y Sapru, S. (2003). Descriptive study of services to LEP students and LEP students with disabilities: Volume I research report. Arlington, VA: Development Associates, Inc.

Wade-Woolley, L. y Siegel, L.S. (1997). The spelling performance of ESL and native speakers of English as a function of reading skill. Reading and Writing: An Interdisciplinary Journal, 9, 387-406.

Wilkinson, G.S. (1993). Wide Range Achievement Test-3. Wilmington, DE: Wide Range, Inc.

Woodcock, R.W. y Johnson, M.B. (1989). Woodcock-Johnson-Revised Tests of Cognitive Ability. Itasca, IL: The Riverside Publishing Company.

Woodcock, R.J., McGrew, K. y Mather, N. (2001). Woodcock-Johnson III Tests of Achievement. Itasca, IL: Riverside.

Recibido: 2 de junio de 2009

Recepción Modificaciones: 19 de agosto de 2009

Aceptado: 22 de agosto de 2009 